\title{
Tratamento cirúrgico concomitante de coarctação de aorta e lesões intracardíacas por esternotomia mediana
}

Marcelo B. JATENE*, David OSTOA*, Carlos A. DIAS*, Arlindo A. RISO*, Miguel BARBEROMARCIAL ${ }^{\star \star}$, Munir EBAID*, Adib D. JATENE*

JATENE, M.B.; OSTOA, D.; DIAS, C.A.; RISO, A.A.; BARBERO-MARCIAL, M.; EBAID, M.; JATENE, A.D. - Tratamento cirúrgico concomitante de coarctação de aorta e lesōes intracardiacas por esternotomia mediana. Rev. Bras. Cir. Cardiovasc., 8(2):130-135,1993.

RESUMO: A associação de lesōes intracardiacas com coarctação de aorta (CoAo) pode ser tratada cirurgicamente em dois tempos, ou em um único procedimento por toracotomia medioesternal, sem que haja, necessariamente, aumento do risco operatório. No periodo de junho de 1988 a dezembro de 1992, 22 crianças (ç̧as) foram operadas através deste acesso cirúgico. A idade variou de 12 dias a 18 anos $(48,9 \mathrm{~m})$, sendo 12 do sexo masculino. Todas apresentavam CoAo de importante repercussão, associada a diferentes lesōes intracardiacas, a saber: CIV em 12 ç̧as (6 isoladas; 3 com estenose mitral e sub-aórtica; 2 com estenose sub-aórtica e 1 com insuficiência mitral);estenose aórtica e sub-aórtica em 5 cças; estenose mitral em 2 cças. Todas as cças foram operadas por toracotomia medioesternal com suporte de circulação extracorpórea e hipotermia profunda, reparaando-se, em primeiro lugar, a CoAo e, em seguida, a lesão intracardíaca. Em 17 cças, a zona coarctada foi ressecada e realizada anastomose término-terminal; em 4, utilizou-se flap de artéria subclávia esquerda e, em 1 cça, a CoAo foi corrigida com interposição de tubo de Dacron. Não foram observados óbitos nesse grupo e as principais complicaçōes imediatas foram síndrome de baixo débito em 3 ç̧as; pneumonia em 2 cças. Em um período de evolução de 1 a $50 \mathrm{~m}$ (17,7 m), 16 ç̧as estāo bem e assintomáticas; 4 apresentaram sintomas dolorosos incaracterísticos e 2 cças tiveram seu seguimento perdido. No mesmo período, após avaliaçāo por ecocardiograma, não se observou CoAo residual. Em conclusão, o tratamento cirúrgico concomitante se mostrou eficiente em cças de diferentes idades e com diferentes lesōes, com baixa morbidade e sem mortalidade no grupo estudado.

DESCRITORES: lesōes cirúrgicas intracardíacas; aorta, coartação, cirurgia.

\section{INTRODUÇĀO}

A coarctação de aorta (CoAo) corresponde a $6,5 \%$ das cardiopatias congênitas, sendo que em cerca de $80 \%$ dos casos ela se apresenta de forma isolada ${ }^{6}$, (associada ou não a canal arterial patente) ou a outras lesões intracardiacas.
O tratamento cirúrgico da CoAo isolada teve seu primeiro relato clínico feito por CRAFOORD \& NYLON ${ }^{4}$ que, em 1944, realizaram ressecção da área coarctada e anastomose término-terminal (ATT). Em 1948, CLAGETT ${ }^{2}$ utilizou a artéria subclávia esquerda, como Blalock descreveu em 8 casos onde a ATT não foi possível. A partir de 1951,

Trabalho realizado no Instituto do Coração do Hospital das Clínicas da Faculdade de Medicina da Universidade São Paulo e no Hospital Sírio-Libanês, São Paulo, SP, Brasil

Apresentado ao $20^{\circ}$ Congresso Nacional de Cirurgia Cardiaca. Maceió, AL, 2 e 3 de abril, 1993.

- Do Instituto do Coração do Hospital das Clinicas da Faculdade de Medicina da Universidade de São Paulo.

- Do Instituto do Coraçăo e do Hospital Sírio-Libanês.

Endereço para separatas: Marcelo B. Jatene. Rua João Moura, 1535. Jardim das Bandeiras. 05412-003 São Paulo, SP, Brasil. 
JATENE, M.B.; OSTOA, D.; DIAS, C.A.; RISO, A.A.; BARBERO-MARCIAL, M.; EBAID, M.; JATENE, A.D. - Tratamento cirúrgico concomitante de coarctação de aorta e lesões intracardíacas por esternotomia mediana. Rev. Bras. Cir. Cardiovasc., $8(2): 130-135,1993$.

LYNXWILLER et alii ${ }^{11}$, KIRKLIN et alii ${ }^{10}$ e MUSTARD et alii ${ }^{14}$ realizaram a mesma operação em crianças com idade inferior a 3 meses. Novas técnicas foram se somando, como as descritas por VORSSCHULTE $19 \mathrm{com}$ a realização de incisão vertical sobre a CoAo e sutura transfersal, e WALDHAUSEN \& NAHRWOLD ${ }^{20}$, que, em 1966, propuseram a correção da CoAo com rotação de um flap de artéria subclávia esquerda. Em nosso meio, MENDONÇA et alii ${ }^{12}$, em 1985, descreveram técnica para correção de CoAo com preservação da irrigação na artéria subclávia esquerda. A utilização de próteses tubulares teve início em 1960, a partir de relato de MORRIS et alii ${ }^{13}$. Todas essas técnicas tinham em comum o mesmo acesso cirúrgico, por toracotomia esquerda látero-posterior.

Entretanto, quando a CoAo está associada a outras lesões intracardíacas passíveis de correção cirúrgica, o tratamento cirúrgico pode ser realizado em 2 tempos ou em único procedimento. Neste caso, o acesso cirúrgico pode variar, podendo se realizar toracotomia esquerda para corrigir a CoAo e, em sequida, reposicionar a criança para tratamento do outro defeito por via mediesternal. BARRATT-BOYES ${ }^{1}$, em 1972, relataram tal procedimento para tratamento de interrupçõ de arco aórtico, CIV e drenagem anômala total de veias pulmonares. Outra opção de tratamento concomitante dos defeitos é por via médico esternal, como descrito por TRUSLER \& ISUKAWA ${ }^{17}$, em 1975 , onde os 2 defeitos são abordados, sendo em primeiro lugar a CoAo e em seguida o defeito intracardíaco, com suporte de circulação extracorpórea (CEC). Um sucessivo número de relatos com esta técnica vem sendo descrito, com variáveis técnicas e táticas, no intuito de buscar uma solução eficiente e definitiva.

A experiência do InCor e do Hospital Sírio-Libanês com esta técnica será descrita a seguir.

\section{CASUISTICA E MÉTODOS}

No período de junho de 1988 a dezembro de 1992, 22 crianças (cças) com idade variando de 12 dias a 18 anos $(48,9 \mathrm{~m})$ foram operadas. Doze $(54,5 \%)$ eram do sexo masculino. Todas as cças eram portadoras de CoAo, que, por parâmetros clínicos e ecocardiográficos, foi considerada de importante repercussão hemodinâmica. $O$ gradiente médio medido pelo ECO foi de $53,5 \mathrm{mmHg}$. Em 6 cças foi realizado cateterismo cardíaco; no auxílio ao diagnóstico. Onze cças apresentavam sintomas de dispnéia, sendo que 1 se encontrava em baixo débito e franca ICC no pré-operatório.

Com relação às lesões intracardíacas associadas à $\mathrm{CoAo}, 12(54,5 \%)$ cças eram portadoras de
CIV, sendo isolada em $6(27,2 \%)$; associada a estenose mitral (EMi) e estonose da via de saída do ventrículo esquerdo em $3(13,6 \%)$; associada a estenose subaórtica (EsubAo) em $2(9,1 \%)$ e em 1 $(4,5 \%)$ associada a insuficiência mitral (IMi). Três $(13,6 \%)$ cças eram portadoras de estenose aórtica (EAo) e $2(9,1 \%)$ de EsubAo. As outras 5 apresentavam os seguintes diagnósticos: atrioventricularis communis forma total; EMi; disfunção de prótese mitral; estenose pulmonar infundíbulo-valvar e transposição das grandes artérias.

Em uma das cças (CoAo + EAo - 18 anos) a CoAo já havia sido operada há 13 anos e houve recoarctação com gradiente de $60 \mathrm{mmHg}$.

Os procedimentos cirúrgicos realizados concomitantemente à correção da CoAo constam na Tabela 1.

TABELA 1

PROCEDIMENTOS CIRÚRGICOS ASSOCIADOS

- Ventriculoseptoplastia comPB

6

- Ventriculoseptoplastia + comiss. Mi + Ressecção EsubAo

- Ressecção anel fibroso subAo

- Comiss Ao

- Ventriculoseptoplastia + plástica V.Mi

- Atrioventriculoseptoplastia

- Comiss. Ao + ressecção subAo

- Plástica V.Mi

- Troca V.Mi - Carbomedics

- Troca V.Ao - Carbomedics

- Aplicação VSVD

- Operação de JATENE

Comiss. - comissurotomia

V.Mi - valva mitral

V.Ao - valva aórtica

VSVD - via de saída do ventriculo direito

\section{Técnica Cirúrgica}

Todas as cças foram operadas por acesso medioesternal e, após pericardiotomia, se realizou o preparo habitual da aorta e das 2 veias cavas para instalação da CEC. Foi feita a dissecção da aorta desde a aorta ascendente, isolando o arco aórtico e seus ramos, bem como o canal arterial ou ligamento arterioso. Toda dissecção foi feita bem próxima à aorta, para evitar lesões em outras estruturas, como nervo frênico e laríngeo-recorrente. Após identificação do canal ou ligamento, procedeu-se a secção e sutura do mesmo (no caso do canal arterial), no sentido de facilitar a mobilização da aorta. Em seguida, após afastamento do tronco pulmonar para a direita e da aurícula esquerda no sentido inferior, procedeu-se à dissecção cuidadosa da 
JATENE, M.B.; OSTOA, D.; DIAS, C.A.; RISO, A.A.; BARBERO-MARCIAL, M.; EBAID, M.; JATENE, A.D. - Tratamento cirúrgico concomitante de coarctaçāo de aorta e lesōes intracardiacas por esternotomia mediana. Rev. Bras. Cir. Cardiovasc. $8(2): 130-135,1993$.

região coarctada, bem como da aorta descendente, distal à CoAo, mobilizando a mesma o suficiente para realização segura da correção da CoAo. Toda essa dissecção é realizada sem CEC. Em $2(9,1 \%)$ cças a correção da CoAo se realizou sem CEC, em função da apresentação favorável após a dissecção. Nesses 2 casos (idades de $13 a$ e $5 a$ 9m com diagnóstico de $\mathrm{CoAo}+$ EsubAo) foi realizada ressecção da zona coarctada e anastomose términoterminal. Em todas as outras $20(90,9 \%)$ a correção da CoAo foi realizada em CEC, sob hipotermia profunda com temperatura entre 16 e 20 graus. Em $18(90 \%)$ utilizou-se hipofluxo em CEC e em $2(10 \%)$, parada circulatória total (idades de $16 \mathrm{~d}$ e $30 \mathrm{~d}$ com diagnóstico de CoAo + CIV) para correção da CoAo.

Após a resolução da CoAo e reperfusão da aorta descendente após desclampeamento, se procedeu à correção do defeito intracardíaco (Tabela 1).

Nas 2 cças em que a CoAo foi operada sem CEC, o defeito intracardíaco foi abordado em CEC com temperatura de 28 graus e normo-fluxo.

Com relação à técnica cirúrgica empregada na correção da CoAo, em $17(77,3 \%)$ ç̧as foi possível a realização com segurança da zona coarctada e ATT, com fio absorvível e sutura contínua em cerca de $70 \%$ dos casos; a idade média nesse grupo foi de $48,5 \mathrm{~m}$. Em $4(18,2 \%)$ cças se utilizou a técnica de Waldhausen, com idade média de $4,2 \mathrm{~m}$. Em uma $(4,5 \%)$ cça de $18 \mathrm{a}$, portadora de ReCoAo + EAo, foi colocado enxerto tubular de Dacron, como bypass com suturas proximal e distal à zona coarctada.

\section{RESULTADOS}

Não houve óbito nesse grupo, com todas as ç̧as tendo recebido alta hospitalar.

O tempo total de CEC variou de $30^{\prime}$ a 139'

As principais complicaçōes imediatas foram HAS em $8(36,3 \%)$ cças; baixo débito cardíaco com necessidade de suporte inotrópico em $2(9,1 \%)$, pneumonia em $2(9,1 \%)$; insuficiência respiratória com necessidade de suporte ventilatório por mais de 48 hs em $2(9,1 \%)$ e arritmia (taquicardia supraventricular) em $1(4,5 \%)$. Houve boa resolução em todos os casos.

Num período de seguimento tardio, que variou de 1 a $50 \mathrm{~m}(17,7 \mathrm{~m}), 18(81,8 \%)$ cças encontramse assintomáticas, sendo 15 sem qualquer medicação e 3 recebendo digoxina e diuréticos em dose proporcional à idade. Quatro $(18,2 \%)$ cças apresentam sintomas incaracterísticos (dores torácicas difusas) mas encontram-se assintomáticas do ponto de vista cardiovascular.
No mesmo período de evolução, o estudo ecocardiográfico não mostrou qualquer evidência de recidiva de $\mathrm{CoAo}$, com ausência de gradiente no local. Os principais achados ecocardiográficos observados foram: EAo residual (gradiente de 45 $\mathrm{mmHg}$ ) com $50 \mathrm{~m}$ de evolução em cça portadora de EAo severa (6 anos de idade na época da operação); IMi e CIV residuais discretos com $42 \mathrm{~m}$ de evolução em cça com diagnóstico de AVC forma total e IAo discreta com $28 \mathrm{~m}$ de evolução em cça de 6 anos portadora de EAo com gradiente préoperatório de $100 \mathrm{mmHg}$.

\section{COMENTÁRIOS}

No início da abordagem terapêtica das cças portadoras de defeitos intracardíacos concomitantes com CoAo, o tratamento de escolha consistia da resolução da CoAo (e eventual cerclagem pulmonar nos casos de CIV) em uma primeira etapa, deixando a correção do defeito intracardíaco para um segundo tempo.

No entretanto, o procedimento inicial nem sempre era isento de risco, sendo que, muitas vezes, o pós-operatório da correção da CoAo era conturbado, em função da existência do defeito intracardíaco. NECHES et alii ${ }^{15}$ confirmam tal afirmação, com relato de alta mortalidade em cças menores de 6 meses de idade, nas quais era feita somente a correção da CoAo e não se abordava a CIV. CONNORS et alii ${ }^{3}$ e HERMANN et alii ${ }^{8}$ relatam alto índice de morbi-mortalidade em cças com abordagem paliativa inicial e posterior correção definitiva. Em função de alguns resultados desfavoráveis, numa segunda etapa, passou-se à abordagem dos 2 defeitos em um único procedimento, através de 2 incisões, sendo a primeira uma toracotomia esquerda para tratar da CoAo e a segunda, toracotomia medioesternal para tratar o defeito associado. KERBER et alii ${ }^{9}$, em 1972, relatam tal procedimento em 2 casos de estenose valvar e supravalvar aórtica associados a CoAo, com bom resultado. BARRATTBOYES et alii ${ }^{1}$ relataram caso de cça portadora de CIV, drenagem anômala de veias pulmonares e interrupção do arco aórtico operada dessa maneira. TIRABOSCHI et alii ${ }^{16}$ mostram bom resultado com esta técnica em 6 ç̧as com idade inferior a 24 meses, portadoras de CIV e CoAo. Dentro desta linha de atuação, em nossa Instituição, foram operadas cças onde se corrigiu, em primeiro lugar, o defeito intracardíaco e, em seguida, a CoAo por 2 incisões diferentes, na maioria casos onde a CoAo era moderada, e se optou pela resolução do defeito principal, para, em seguida, já em situação clínica mais favorável, corrigir a CoAo.

A partir de 1988 , em nosso grupo, todas as cças 
JATENE, M.B.; OSTOA, D.; DIAS, C.A.; RISO, A.A.; BARBERO-MARCIAL, M.; EBAID, M.; JATENE, A.D. - Tratamento cirúrgico concomitante de coarctação de aorta e lesōes intracardiacas por esternotomia mediana. Rev. Bras. Cir. Cardiovasc., $8(2): 130-135,1993$.

com cardiopatias associadas a CoAo passaram a ser operadas em único procedimento, através de uma única incisão, com importante redução do tempo cirúrgico e resolução definitiva dos 2 defeitos. TRUSLER \& ISUKAWA 17 mostravam desde 1975 tal opção cirúrgica como viável quando da correção de CIV e interrupção do arco aórtico em cça de 13 dias de vida. DE LEON et alii ${ }^{5}$, UNGERLEIDER \& ERBERT ${ }^{18}$ e HEINEMANN et alii ${ }^{7}$ também mostraram a cirurgia concomitante dos dois defeitos como primeira opção terapêutica para estes casos.

Uma eventual limitação técnica para a correção da CoAo por via mediosternal seria a impossibilidade da aplicação de alguma das técnicas cirúrgicas de que se dispõe, em função da dificuldade de acesso, já que o campo cirúrgico fica restrito e profundo, com estruturas nobres se sobrepondo à região a ser abordada. No entanto, fazendo-se dissecção cuidadosa, afastando-se tronco pulmonar, aurícula esquerda e pulmão esquerdo, o acesso fica muito facilitado. Em nossa experiência, 17 (77,3\%) ç̧as tiveram a CoAo ressecada com realização de ATT. Tal técnica exige que a aorta esteja totalmente liberada e que a a orta descendente possa ser amplamente mobilizada no sentido cranial, o que se pode ser feito sem dificuldade por via mediana, com bons resultados imediatos e a longo prazo. Em 4 $(18,2 \%)$ cças se utilizou a técnica de Waldhausen e em $1(4,5 \%)$ cça foi utilizado tubo de Dacron para correção da CoAo. DE LEON et alii ${ }^{5}$ operaram 5 cças com ressecção da CoAo e ATT por via mediana, enquanto que UNGERLEIDER \& ERBERT 18 preferiram a utilização de patches de diferentes materiais para correção da CoAo em 12 cças; nesse caso, a dissecção e a liberação da aorta podem ser mais econômicas. HEINEMANN et alii 7 mostraram experiência de 8 casos com ressecção e ATT.

Em nossa experiência, as cças menores, em especial os neonatos, apresentaram maior facilidade para correção da CoAo, em função da menor profundidade do tórax e do fato de que as estruturas vasculares são mais elásticas e, portanto, mais facilmente mobilizáveis; em especial, a realização da ATT fica mais facilitada.

Ainda sob o aspecto técnico, não se observou maior dificuldade na revisão das linhas de sutura da anastomose na aorta. $\mathrm{Na}$ abordagem da face anterior da aorta, não se observaram maiores problemas, bastando afastar as estruturas vizinhas e expor a zona da antiga CoAo. A vizibilização da face posterior da aorta é possível mobilizando-se a aorta no sentido anterior, a partir da região da croça, e com pequena manobra de rotação se expõe a sutura posterior, revisando-a com cuidado.

Com relação à CEC, em $20(90,1 \%)$ cças de nosso estudo, a correção da CoAo se fez em CEC, sob hipotermia profunda com temperatura entre 16 e 20 graus. Em 2 cças a abordagem da CoAo foi feita sem CEC, face à situação anatômica favorável. DE LEON et alii ${ }^{5}$ relatam cça onde a correção da CoAo foi feita sem CEC, sendo as demais em CEC e hipotermia profunda. A mesma conduta é descrita por HEINEMANN et alii ${ }^{7}$ em 8 cças. UNGERLEIDER \& ERBERT 18 realizaram correção da CoAo sob parada circulatória total em $50 \%$ das ç̧as, num total de 12 cças.

Em conclusão, o tratamento cirúrgico concomitante da CoAo e defeitos intracardíacos pode ser realizado com segurança, com baixo índice de morbi-mortalidade, em cças com idades variáveis. Não se observou limitação no uso de alguma das técnicas cirúrgicas habituais. Os resultados apresentados confirmam a utilização do procedimento combinado como opção inicial para o tratamento desse tipo de lesão. 
JATENE, M.B.; OSTOA, D.; DIAS, C.A.; RISO, A.A.; BARBERO-MARCIAL, M.; EBAID, M.; JATENE, A.D. - Tratamento cirúrgico concomitante de coarctaçăo de aorta e lesōes intracardiacas por esternotomia mediana. Rev. Bras. Cir. Cardiovasc., $8(2): 130-135,1993$.

\section{RBCCV 44205-208}

GJATENE, M. B.; OSTOA, D.; DIAS, C. A.; RISO, A. A.; BARBERO-MARCIAL, M.; EBAID, M.; JATENE, A. D. - Coarctation of the aorta and associated intracardiac lesions: concomitant repair with an anterior midline approach. Rev. Bras. Cir. Cardiovasc., 8(2):130-135, 1993.

ABSTRACT: The association of intracardiac lesions with coarctation of the aorta ( $\mathrm{CoAO})$ is a situation that could be repaired in only one surgery by anterior midline approach, despite of sugestion of increased risk if compared with two stage repair. Our experience with this approach is a group of 22 children, with ages ranging from $12 \mathrm{~d}$ to $18 \mathrm{y}(48.9 \mathrm{~m})$, being 12 male, from Jun 88 to Dec 92 . All children had CoAo associated whith different intracardiac lesions like VSD in 12 (isolated in 6; with mitral and subaortic stenosis in 3; with subaortic stenosis in 2 and 1 with mitral regurgitation); aortic and subaortic stenosis in 5 , mitral stenosis in 2. All children were operated on by anterior midline approach using cardiopulmonary bypass and profound hypothermia to repair first the CoAo and after the intracardiac lesion. In 17 children the CoAo zone was excised with end to end anastomosis; in 4 the left subclavian flap was used and in 1 a Dacron graft was used. No deaths were observed and the main immediate complications were low cardiac output syndrome in 3 and pneumonia in 2 children. In a follow up period from 1 to $50 \mathrm{~m}(17.7 \%), 16$ children are well and assymptomatic and 4 have incaracteristic chest pain. In the same period, the CoAo repair was evaluated by ECHO with no residual stenosis. In conclusion, the concomitant repair showed good results with low morbidity and no mortality in children with different ages and diagnosis.

DESCRIPTORS: intracardiac surgical lesions; aortic coarctation, surgery.

\section{REFERÊNCIAS BIBLIOGRÁFICAS}

1 BARRTTT-BOYES, B. G.; NICHOLLS, T. T.; BRANDTT, P. W. T.; NEUTZE, J. M. - Aortic arch interruption associated with patent ductus arteriosus, ventricular septal defect, and total anomalous pulmonary venous correction. J. Thorac. Cardiovas. Surg., 63: 367$373,1972$.

2 CLAGETT, O.T. - The surgical treatment of coarctation of the aorta. Mayo Clin. Proc., 23: 359-360, 1948.

3 CONNORS, J. P.; HARTMANN, A. F.; WELDON, C. S. - Considerations in the surgical management of infantile coarctation of aorta. Am. J. Cardiol., 38: 489-492, 1978.

4 CRAFOORD, C, \& NYLIN, G. - Congenital coarctation of the aorta and its surgical treatment. J. Thorac. Surg., 14: 347-361, 1945.

5 DeLEON, S. Y.; IDRISS, F. S.; ILBAWI, M. M.; TIN, N.; BERRY, T. - Transmediastinal repair of complex coarctation and interrupted aortic arch. J. Thorac. Cardiovasc. Surg., 82: 98-102, 1991.

FYLER, D. C. - Report of the New England Regional Infant Cardiac Program. Pediatrics, 64 (Supl.): 377. $461,1980$.

7 HEINEMANN, M.; ZIEMER, G.; LUHMER, I.; HAVERICH, A.; KALLFELZ, H.C.; BORST, H.G. - Coarctation of the aorta in complex congenital heart disease: simultaneous repair via sternotomy. Eur. J. CardioThorac. Surg., 4: 482-486, 1990.
HERMANN, V. M.; LAKS, H.; FAGAN, L.; TERSCHLUSE, D.; WILLMAN, V.L. - Repair of aortic coarctation in the first year of life. Ann. Thorac. Surg., 25: 57-63, 1978.

9 KERBER, R.E., GREENE, R. A.; COHN, L. H., WEXLER, L.; KRISS, J. P.; HARRISON, D. C. - Multiple left ventricular outflow obstructions: aortic valvular and supravalvular estenoses and coarctation of the aorta. J. Thorac. Cardiovasc. Surg., 3: 374-379, 1972.

10 KIRKLIN, J. W.; BURCHELL, H. B.; PUCH, D. G.; BURKE, E. C.; MILLS, S. D. - Surgical treatment of coarctation of the aorta in a ten-week-old infant: report of a case. Circulation, 6: 411-414, 1952.

11 LYNXWILER, C. P.; SMITH, S.; BABICH, J. - Coarctation of the aorta: report of a case. Arch. Pediatr., 68: 203-207, 1951.

12 MENDONÇA, J. T.; CARVALHO, M. R.; COSTA, R. K.; FRANCO FILHO, E. - Coarctation of the aorta: a new surgical technique. J. Thorac. Cardiovasc. Surg., 90: 445-447, 1985.

13 MORRIS, G. C.; COOLEY, D. A.; DeBAKEY, M. E.; CRAWFORD, E. S. - Coarctation of the aorta with particular amphasis upon improved techniques of surgical repair. J. Thorac. Cardiovasc. Surg., 40: 705-709, 1960.

14 MUSTARD, W. T.; ROWER, R. D.; KETIH, J. D.; SIREK, A. - Coarctation of the aorta with special reference to the first year of life. Ann. Surg., 141: 429-436, 1955. 
JATENE, M.B.; OSTOA, D.; DIAS, C.A.; RISO, A.A.; BARBERO-MARCIAL, M.; EBAID, M.; JATENE, A.D. - Tratamento cirúrgico concomitante de coarctação de aorta e lesōes intracardíacas por esternotomia mediana. Rev. Bras. Cir. Cardiovasc., 8(2):130-135,1993.

15 NECHES, W. H.; PARK, S. C.; LENOX, C. C.; ZUBERBUHLER, J. R.; SIEWERS, R. D.; HARDESTY, R. L. - Coarctation of the aorta with ventricular septal defect. Circulation, 55: 189$194,1977$.

TIRABOSCHI, R.; ALFIERI, O.; CARPENTIER, A.; PARENZAN, L. - One stage correction of coarctation of the aorta associated with intracardiac defects in infancy. J. Cardiovasc. Surg., 19: $11-16,1978$.

17 TRUSLER, G.A. \& ISUKAWA, T. - Interrupted aortic arch and ventricular septal defect: direct repair through a median sternotomy incision in a 13-day- old infant. J. Thorac. Cardiovasc. Surg., 69: 126131, 1975.

18 UNGERLEIDER, R. M, \& ERBERT, P. A. - Indications and techniques for midline approach to aortic coarctation in infants and children. Ann. Thorac. Surg., 44: 517-522, 1987.

19 VORSSCHULTE, K. - Surgical corretion of the aorta by an "isthmus plastic" operation. Thorax, 16: 338 $345,1961$.

20 WALDhAuSEN, J. A. \& NAHRWOLD, D. L. - Repair of coarctation of the aorta with a subclavian flap. J. Thorac. Cardiovasc. Surg., 51: 532-533, 1966. 